\section{Tendência da epidemia de AIDS em usuários de drogas injetáveis no Município de São Paulo de 1985 a $1997^{*}$}

\section{Trend of the AIDS epidemic in intravenous drug users in the Municipality of São Paulo from} 1985 to 1997

Maria do Socorro Castelo Branco de Oliveira Bastos

Concluinte do Curso de Mestrado da Faculdade de Saúde Pública

Maria do Rosário Dias de Oliveira Latorre

Departamento de Epidemiologia

Faculdade de Saúde Pública

Universidade de São Paulo

\section{Eliseu Alves Waldman, MD, PhD²}

Departamento de Epidemiologia

Faculdade de Saúde Pública

Universidade de São Paulo

Correspondência para/Correspondence to:

Av. Dr. Arnaldo 715

01246-904 -São Paulo, SP

e-mail: eawaldma@usp.br

\section{Auxílio financeiro}

Conselho Nacional de Desenvolvimento Técnico e Científico (CNPq) (bolsa de mestrado e de pesquisa - Processo no 30-0318/97-9)

*Baseado na Dissertação de Mestrado: Tendência da Epidemia de AIDS em Usuários de Drogas Injetáveis no Município de São Paulo de 1985 a 1997

\section{Resumo}

Objetivo. Analisar a tendência da epidemia de AIDS em adultos, no período de 1985 a 1997, no Município de São Paulo, focalizando principalmente os usuários de drogas injetáveis (UDI), considerando o grande impacto da epidemia nesse grupo populacional. Metodologia. A fonte dos dados foi a vigilância de AIDS do Estado de São Paulo. O método estatístico utilizado para a análise de tendência temporal dos 8.558 casos de AIDS entre UDI, e dos 16.756 entre não UDI, foi a regressão polinomial.

Resultados. No período de 1985 a 1992 a tendência foi de ascensão para os casos de AIDS entre UDI e não UDI a partir desse ponto e, observou-se um declínio para UDI e manutenção em platô elevado para os não UDI, em conseqüência do crescimento constante do número de casos entre as mulheres e homens heterossexuais. Os coeficientes de regressão ajustados $\left(\mathrm{R}_{\mathrm{a}}{ }_{\mathrm{a}}\right)$ de todos os modelos ficaram acima de $70 \%$, e os modelos que melhor explicaram as tendências foram os de segunda ordem (parábola), exceto para heterossexuais não UDI cuja tendência foi de aumento linear.

Conclusão. No final do período considerado, os dados sugerem um declínio da epidemia em UDI, a estabilização em níveis elevados para o total de não UDI, e a manutenção da tendência de crescimento da epidemia para pessoas heterossexuais não UDI.

Palavras-chave: Síndrome de imunodeficiência adquirida, tendências. Síndrome de imunodeficiência adquirida, epidemiologia. Abuso de substâncias por via endovenosa. 
Abstract

Objective. To analyze trends of AIDS epidemic among adults, from 1985 to 1997, in the city of São Paulo, focussing primarily on intravenous drug users (IDU), owing to the major impact of the epidemic on this population group.

Methodology. Data from the AIDS surveillance of São Paulo State were analyzed. The statistical method applied to analyze the time series of the 8,558 cases of AIDS among IDU and of the 16,756 cases among non-IDU was polynomial regression.

Results. From 1985 to 1992 there was a increase in AIDS cases both in IDU and nonIDU. Thereafter there was a decrease in cases among IDU, while cases among non-IDU leveled off owing to the constant growth trend among heterosexual men and women. Adjusted regression coefficients $\left(\mathrm{R}_{\mathrm{a}}^{2}\right)$ for all models remained higher than $70 \%$, and the second-order (parabolic) models explained better the trends, except in the case of heterosexual non-IDU, in which the model was linear.

Conclusion. At the end of the period under study the data suggest a decrease in the epidemic among IDU, while the trend among non-IDU keep flat at high level, and showed a constant increase among heterosexual.

Keywords: Acquired immunodeficiency syndrome, trends. Acquired immunodeficiency syndrome, epidemiology. Substance abuse, intravenous.

\section{Introdução}

A epidemia de AIDS no Brasil totalizou 197.773 casos notificados até o final de 1999, sendo que $47,7 \%$ e $21,4 \%$ desse total eram residentes, respectivamente, no Estado de São Paulo e no município da capital ${ }^{1,2}$. A partir do final do anos 80, os usuários de drogas injetáveis (UDI) passaram a ter participação importante na epidemia, atingindo em 1991 $25,9 \%$ dos casos notificados no país ${ }^{1}$. Os estudos disponíveis a respeito da soroprevalência de infecção por HIV entre UDI no Brasil apontam níveis altos, ${ }^{3-7}$ mostrando a importância do acompanhamento desse grupo em virtude do elevado risco de infecção.

A vulnerabilidade dos UDI à infecção por $\mathrm{HIV}^{8-10}$, assim como a outras doenças infecciosas $^{3,9,11,12,}$ resulta do comportamento sexual e do hábito de compartilhar seringas. Por sua vez, a transmissão sexual do HIV de UDI para não UDI foi fator relevante na disseminação da epidemia de AIDS $^{13,14}$.

O presente texto buscará construir uma imagem da epidemia de AIDS no Município deSão Paulo, com enfoque principal nos UDI, dada a importância que têm tido para a dinâmica da AIDS na cidade e a necessidade de tornar visível o impacto da epidemia nesse grupo, fornecendo subsídios para a eliminação dos obstáculos legais a algumas propostas preventivas e também diminuir preconceitos existentes na sociedade.

O estudo focalizará a cidade de São Paulo, pela suas características de grande metrópole, por ter apresentado uma das mais elevadas taxas de morbi-mortalidade por AIDS no país, por ser apontada como principal centro difusor da epidemia ${ }^{15}$ e pelo contingente de mais de 6 milhões de adultos potencialmente expostos.

O objetivo dessa investigação é analisar a tendência temporal da epidemia de AIDS em UDI e em não UDI, no Município de São Paulo, durante o período de 1985 a 1997, segundo idade e sexo. 


\section{Metodologia}

\section{População de Estudo}

Foram analisados os casos de AIDS, em pessoas com 15 anos ou mais, notificados até 30 de setembro de 1998 ao Sistema de Vigilância Epidemiológica (SVE) da Secretaria de Saúde de São Paulo, com data do diagnóstico entre 1985 e 1997 e local de residência no Município de São Paulo.

Para a definição de caso foram utilizados os critérios de $1985^{16}, 1987^{17}, 1992^{18}$, Rio de Janeiro/Caracas, CDC modificado e excepcional, de 1996, de Óbitos presumidos de AIDS $^{19}$, além da revisão de $1998^{20}$.

Do total de 32.351 casos notificados no período de estudo foram excluídos 7.044 $(22,0 \%)$, dos quais 6.476 por ser ignorada a categoria de exposição, 437 por apresentarem a transfusão de sangue e hemoderivados como única exposição de risco, 128 por ausência da data do nascimento e 7 por não existir informação do ano do diagnóstico.

Comparando as características dos ca- sos excluídos por ausência de informação relativa à categoria de exposição (20,1\%) com aqueles efetivamente estudados, não foi observada diferença estatisticamente significativa em relação ao sexo $(\mathrm{p}=0,080)$, mas houve diferenças significativas $(\mathrm{p}<0,001)$ entre as médias das idades e o número de casos por ano. Porém, não foram consideradas relevantes para a análise, pois a diferença entre as médias das idades foi de apenas um ano e porque, provavelmente, a definição posterior da população estudada, por categoria de exposição, se dará de maneira homogênea.

O Sistema de Vigilância da AIDS utiliza o banco de dados do Sistema de Informação de Agravos de Notificação (SINAN) que categoriza os casos de AIDS de maneira múltipla, associando o tipo de relação sexual, a hemofilia, transfusão e uso de droga injetável. No presente estudo os casos confirmados foram reclassificadas quanto à categoria de exposição na forma apresentada no Quadro 1. Todas as categorias têm casos associados a hemofilia ou transfusão, com

Quadro 1 - Categorias utilizadas no estudo a partir das categorias existentes no SINAN (Sistema de Informação de Agravos de Notificação).

Chart 1 - Category of risk exposure applied in this study according to AIDS case classification criteria used by the São Paulo State AIDS surveillance

\begin{tabular}{|ll|}
\hline Categorias do Estudo' & Categorias do SINAN \\
\hline UDI heterossexual & $\begin{array}{l}\text { Exposição heterossexual + UDI ou UDI + relação sexual com } \\
\text { sexo oposto ou ignorada a parceria }{ }^{2}\end{array}$ \\
\hline Não UDI heterossexual & $\begin{array}{l}\text { Exposição heterossexual + não UDI ou heterossexual + uso de } \\
\text { drogas ignorado }\end{array}$ \\
\hline UDI homossexual & $\begin{array}{l}\text { Exposição homossexual ou bissexual + UDI ou UDI + parceria } \\
\text { sexual do mesmo sexo }\end{array}$ \\
\hline Não UDI homossexual & $\begin{array}{l}\text { Exposição homossexual ou bissexual + não UDI ou uso de } \\
\text { drogas ignorado }\end{array}$ \\
\hline
\end{tabular}

Notas:

1- Todas as categorias têm casos associados a hemofilia ou transfusão, com proporção maior para heterossexuais, estatisticamente significativa $(\mathrm{p}<0,001)$, em relação aos HSH, mas não significativa entre UDI e não UDI.

2- Definiu-se esse critério ao notar-se que os coletores de dados estabelecem uma hierarquia, priorizando as categorias homossexual ou bissexual, seguida da associação ou não a uso de droga injetável, para só então classificar o caso como UDI. Somente quando todas essas categorias não permitem classificar o caso, passam a procurar a exposição por contato heterossexual, portanto, deixando aparentemente de informar para os UDI a prática heterossexual, mas não a homossexual ou bissexual.

Notes:

1. All categories of AIDS cases associated with hemophilia and blood transfusion, with higher proportion for heterosexuals individuals, statistically significant $(p<0,001)$ in relation to MSM, but not statistically significant for IDU and non-IDU.

2.These criteria were defined in order to follow the hierarchy established by AIDS surveillance to classify AIDS cases as associated with injecting drug use. Only when all these categories do not allow the case to be classified, is heterosexual contact sought. Therefore, apparently, heterosexual practice for IDU is not informed, differently from homosexual or bisexual contact 
proporção maior para heterossexuais, estatisticamente significativa ( $p<0,001)$, em relação aos homens que fazem sexo com homens (HSH), mas não significativa entre UDI e não UDI.

\section{Procedimentos para Análise de Tendência}

A análise de tendência relativa ao período de 1985 a 1997 foi realizada utilizando-se modelos de regressão polinomiais para séries históricas, sendo a variável dependente (Y) o número de casos de AIDS em pessoas com 15 anos e mais, e a variável independente $(\mathrm{X}) \mathrm{o}$ ano.

No início foram analisados os diagramas de dispersão, gerados pelo software SPSS for Windows 8.0 $0^{21}$, entre o número de casos e os anos de estudo, para observar sua relação. Em seguida, com auxílio do mesmo software, foi realizada a modelagem pela técnica de regressão, partindo-se de modelo mais simples, como o linear $\left(Y=\beta_{0}+\beta_{1} X\right)$, testandose, em seguida, modelos mais complexos, como o de segundo grau $\left(Y=\beta_{0}+\beta_{1} X+\beta_{2} X^{2}\right)$, terceiro grau $\left(Y=\beta_{0}+\beta_{1} X+\beta_{2} X^{2}+\beta_{3} X^{3}\right)$ e exponencial $\left(Y=e^{\beta 0+\beta 1 X}\right)$. Para evitar a autocorrelação entre os pontos, a variável tempo foi centralizada através do ponto médio da série histórica ${ }^{22}$. A técnica foi descrita por Kleinbaum e colaboradores ${ }^{23}$.

A escolha do melhor modelo foi baseada no nível de significância (p) e na análise do resíduo, preenchendo as suposições de independência e variância constante dos erros. A significância estatística da tendência foi admitida quando o modelo obteve $\mathrm{p}<0,050$.

Latorre $^{22}$, que utiliza o método de regressão para análise de tendência temporal de câncer de estômago no Brasil, avalia que a vantagem do modelo de regressão sobre os demais é sua facilidade de elaboração e interpretação dos seus resultados, além de grande poder estatístico.

Foram elaborados modelos para UDI e não UDI, para o total de casos, segundo sexo e categoria de exposição, com uma média de 3 modelos para cada categoria de análise, totalizando 30 modelos dos quais foram selecionados 10 .

Foram gerados 3 modelos para 10 faixas etárias, em ambos os sexos, para UDI e não UDI, totalizando 120 modelos dos quais foram selecionados 32 modelos. Os casos de AIDS em UDI foram agrupados em duas faixas etárias para a elaboração de modelos, divididos por sexos, sendo que, dos 12 modelos gerados, foram escolhidos 4 . Os casos de AIDS em homens não UDI foram agrupados em 3 faixas etárias, o que originou 9 modelos, dos quais foram selecionados 3 .

\section{Resultados}

O número total de casos de AIDS analisados foi de 25.314 (Tabela 1), dos quais 33,8\% eram constituídos por UDI; ao longo da série esta porcentagem chegou a quase $42 \%$ em 1991, passando a decair desde então.

As curvas de tendência da epidemia entre UDI e não UDI foram inicialmente semelhantes, crescentes até 1992. A partir de 1993, no entanto, apresentaram-se distintas: a de não UDI apresentou discreta queda e estabilização emníveis elevados e a de UDI um declínio mais acentuado (Figura 1-A). Os modelos foram estatisticamente significativos ( $\mathrm{p}<0,001)$, com $\mathrm{R}^{2}$ de $95 \%$ para não UDI e de $85 \%$ para UDI.

A exposição através de contato sexual com pessoas do mesmo sexo apresentou, para UDI e não UDI, curvas de tendência semelhantes e crescentes até 1992, passando a decrescer a partir desse ponto (Figura 1-B). Os modelos foram estatisticamente significativos, com $\mathrm{R}^{2}$ de $89 \%$ para não UDI e $85 \%$ para UDI.

A tendência da epidemia para pessoas cuja categoria de exposição foi relações heterossexuais apresentou curva crescente até 1992 entre os UDI, com diminuição da velocidade a partir desse ponto. Entre os não UDI houve elevação constante no tempo, com aumento médio de 107 casos por ano (Figura 1-C). Entre 1993 e 1994 a curva referente aos não UDI suplantou a de UDI. Os dois modelos foram estatisticamente significativos, com $\mathrm{R}^{2}$ a de $84 \%$ para UDI e $95 \%$ para não UDI. 
Tabela 1 - Distribuição dos casos de AIDS em UDI e não UDI, na faixa etária de 15 anos ou mais, segundo período de 1985 a 1997 no Município de São Paulo

Table 1 - AIDS cases among IDU and non-IDU in the 15 year-old or more age group. 1985-1997. City of São Paulo

\begin{tabular}{lrrrrrr}
\hline Ano & \multicolumn{2}{c}{ Não UDI } & \multicolumn{2}{c}{ UDI } & \multicolumn{2}{c}{ Total } \\
& № & $\%$ & № & $\%$ & № & $\%$ \\
\hline 1985 & 200 & 91.7 & 18 & 8.3 & 218 & 100.0 \\
1986 & 314 & 84.9 & 56 & 15.1 & 370 & 100.0 \\
1987 & 573 & 78.7 & 155 & 21.3 & 728 & 100.0 \\
1988 & 850 & 72.5 & 323 & 27.5 & 1173 & 100.0 \\
1989 & 1013 & 64.1 & 567 & 35.9 & 1580 & 100.0 \\
1990 & 1323 & 58.9 & 923 & 41.1 & 2246 & 100.0 \\
1991 & 1527 & 58.3 & 1093 & 41.7 & 2620 & 100.0 \\
1992 & 1985 & 61.9 & 1221 & 38.1 & 3206 & 100.0 \\
1993 & 1830 & 63.6 & 1046 & 36.4 & 2876 & 100.0 \\
1994 & 1781 & 66.7 & 891 & 33.3 & 2672 & 100.0 \\
1995 & 1718 & 67.4 & 831 & 32.6 & 2549 & 100.0 \\
1996 & 1821 & 69.5 & 801 & 30.5 & 2622 & 100.0 \\
1997 & 1821 & 74.2 & 633 & 25.8 & 2454 & 100.0 \\
Total & 16756 & 66.2 & 8558 & 33.8 & 25314 & 100.0 \\
\hline
\end{tabular}

O número de casos entre mulheres sempre foi inferior ao dos homens. Dos 25.314 casos selecionados para o estudo, $13,5 \%$ (3.414) eram mulheres não UDI e 5,6\% (1.406) mulheres UDI. As tendências da epidemia nesses dois grupos mostraram-se distintas desde o início; entre as UDI houve crescimento de 1986 até 1992, com queda a partir desse ponto. Por sua vez, houve crescimento constante da epidemia entre as mulheres não UDI, com um aumento médio anual de 58,7 casos (Figura 1-D). Os modelos foram estatisticamente significativos, com $\mathrm{R}^{2}{ }_{\mathrm{a}} \mathrm{de}$ $81 \%$ para UDI e de $95 \%$ para não UDI.

Foram 13.342 os casos atingindo homens não UDI, sendo que $73 \%$ deles eram homens que fazem sexo com homens (HSH), enquanto os homens UDI abrangiam 7.152 casos, $80 \%$ deles pertenciam ao grupo de heterossexuais. As tendências da epidemia para o sexo masculino, em UDI e não UDI, foram bastante semelhantes, com crescimento até 1992 e decrescendo a partir de 1993 (Figura 1-E). O R ${ }_{a}^{2}$ foi de $93 \%$ para não UDI e $84 \%$ para UDI, e os modelos foram significativos do ponto de vista estatístico.

Para maior compreensão dessas tendên- cias, realizou-se a análise por faixa etária, segundo sexo, entre UDI e não UDI (Tabela 2). A modelagem para os casos de AIDS entre UDI pôde ser realizada para a maioria das faixas etárias, exceto para mulheres na faixa etária de 45 anos ou mais e homens na faixa etária de 55 anos e mais, devido à pequena quantidade e/ou a inexistência de casos em vários anos da série.

Para os casos de AIDS entre não UDI, apenas os modelos para a faixa etária de 15 a 19 anos não foram estatisticamente significativos, seja em homens ou mulheres, mostrando que a epidemia para os não UDI nesta faixa etária esteve estável entre 1985 a 1997.

As tendências dos casos de AIDS entre mulheres UDI, para todas as faixas etárias à exceção de uma, foram semelhantes: crescentes no início da epidemia, passando a decair em anos mais recentes. Apenas na faixa etária de 35 a 39 anos a tendência foi de crescimento constante durante todo o período (2,5 casos ao ano).

As tendências dos casos de AIDS entre mulheres não UDI mostraram crescimento constante em todas as faixas etárias, sempre superando numericamente as mulheres 


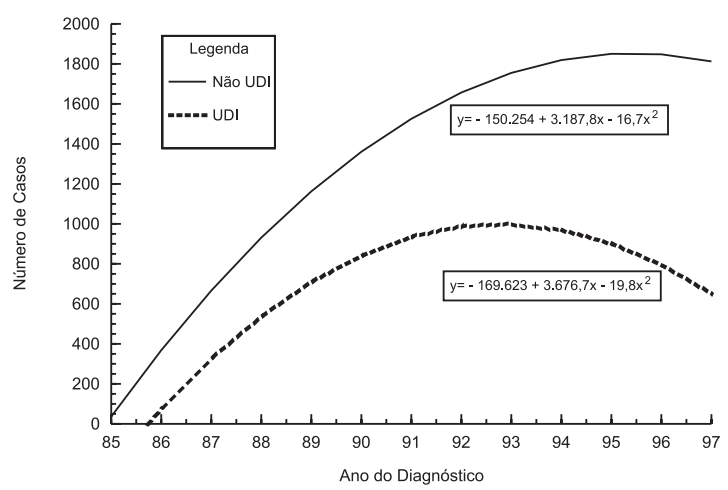

(A)

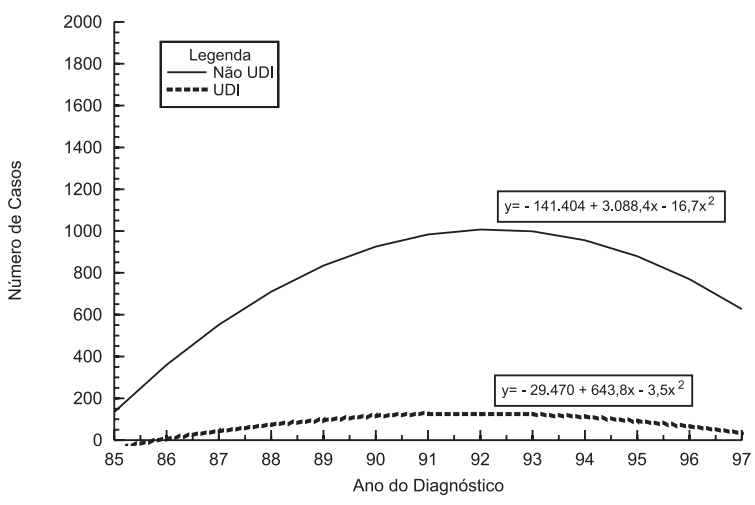

(B)

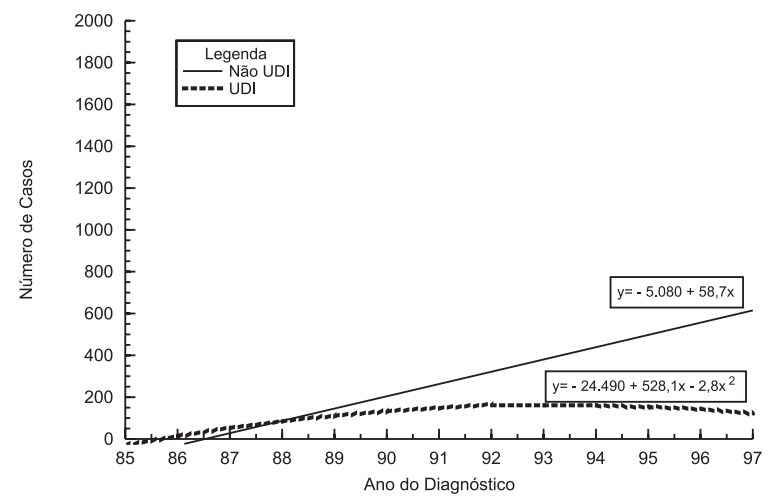

(D)

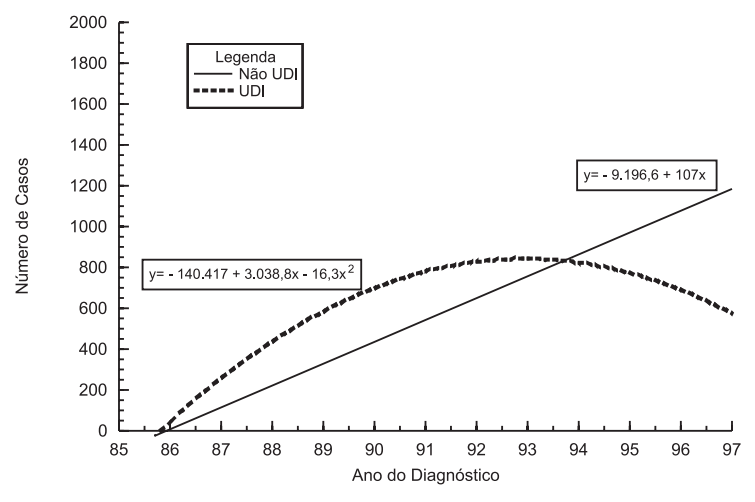

(C)

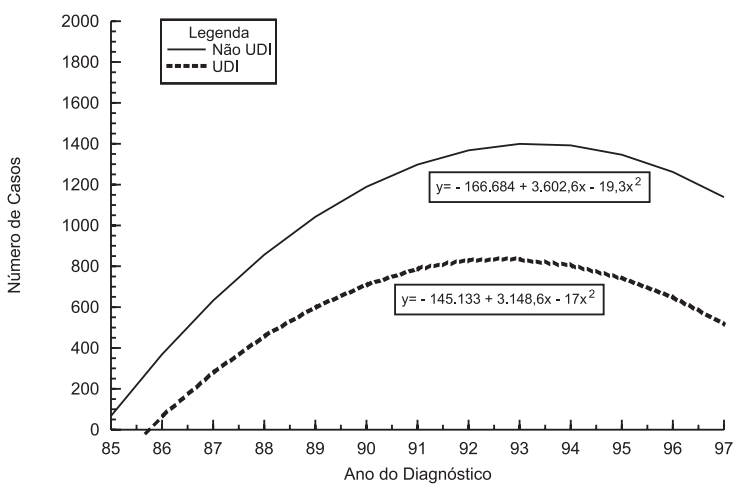

$(\mathrm{E})$

Figura 1 - Tendência dos casos de AIDS em pessoas UDI e não UDI (A); entre homens que fazem sexo com homens (HSH) (B); pessoas com práticas heterossexuais (C); mulheres (D); e homens (E) - Município de São Paulo - 1985 a 1997. Figure 1 - Trend of AIDS cases among IDU and non-IDU (A); MSM (B); heterosexual individuals (C); woman (D); men (E). City São Paulo- 1985-1997. 
Tabela 2 - Modelos de regressão dos casos de AIDS, por sexo e faixa etária em UDI e não UDI - Município de São Paulo - 1985 a 1997.

Table 2 - Regression models of AIDS cases, by gender and age group. City of São Paulo - 1985-1997.

\begin{tabular}{|c|c|c|c|c|c|c|c|}
\hline \multirow{2}{*}{$\begin{array}{l}\text { Faixa Etária } \\
\text { (anos) }\end{array}$} & \multirow{2}{*}{ Sexo } & \multicolumn{3}{|l|}{ UDI } & \multicolumn{3}{|l|}{ Não UDI } \\
\hline & & Modelo* & $\mathrm{R}_{\mathrm{a}}^{2}(\%)$ & $\mathrm{p}$ & Modelo* & $\mathrm{R}_{\mathrm{a}}^{2}(\%)$ & $\mathrm{p}$ \\
\hline \multirow[t]{2}{*}{$15-19$} & Feminino & $y=-1.662,3+36,5 x-0,2 x^{2}$ & 63 & 0,002 & $y=-49,8+0,5 x$ & 37 & 0.016 \\
\hline & Masculino & $y=-9.810,8+217,1 x-1,2 x^{2}$ & 62 & 0,003 & $y=-693,7+15,2 x-0,8 x^{2}$ & 6 & 0.284 \\
\hline \multirow[t]{2}{*}{$20-24$} & Feminino & $y=-7.874,1+172,2 x-0,9 x^{2}$ & 78 & 0,001 & $y=-534,2+6,2 x$ & 82 & $<0,001$ \\
\hline & Masculino & $y=-38.214,0+839,3 x-4,6 x^{2}$ & 73 & $<0,001$ & $y=-8.691,3+190,5 x-1,0 x^{2}$ & 72 & $<0,001$ \\
\hline \multirow[t]{2}{*}{$25-29$} & Feminino & $y=-7.867+170,2 x-0,9 x^{2}$ & 65 & 0,002 & $y=-1.206,2+13,9 x$ & 94 & $<0,001$ \\
\hline & Masculino & $y=-42.632,0+924,9 x-4,9 x^{2}$ & 83 & $<0,001$ & $y=-30.790,0+667,6 x-3,6 x^{2}$ & 91 & $<0,001$ \\
\hline \multirow[t]{2}{*}{$30-34$} & Feminino & $y=-4.568,4+97,6 x-0,5 x^{2}$ & 74 & $<0,001$ & $y=-1.165,8+13,4 x$ & 93 & $<0,001$ \\
\hline & Masculino & $y=-31.241,0+672,8 x-3,6 x^{2}$ & 87 & $<0,001$ & $y=-35.089,0+753,9 x-4,0 x^{2}$ & 91 & $<0,001$ \\
\hline \multirow[t]{2}{*}{$35-39$} & Feminino & $y=-211,1+2,5 x$ & 78 & $<0,001$ & $y=-813,0+9,4 x$ & 91 & $<0,001$ \\
\hline & Masculino & $y=-14.485,0+309,7 x-1,6 x^{2}$ & 85 & $<0,001$ & $y=-32.974,0+710,9 x-3,8 x^{2}$ & 92 & $<0,001$ \\
\hline \multirow[t]{2}{*}{$40-44$} & Feminino & $y=-122,+1,4 x$ & 90 & $<0,001$ & $y=-579,8+6,7 x$ & 90 & $<0,001$ \\
\hline & Masculino & $y=-5.820,0+123,1 x-0,6 x^{2}$ & 77 & $<0,001$ & $y=-21.474,0+462,9 x-2,5 x^{2}$ & 86 & $<0,001$ \\
\hline \multirow[t]{2}{*}{$45-49$} & Feminino & $\ldots$ & & & $y=-302,0+3,5 x$ & 87 & $<0,001$ \\
\hline & Masculino & $y=-187,5+2,2 x$ & 75 & $<0,001$ & $y=-17.957,0+388,6 x-2,1 x^{2}$ & 87 & $<0,001$ \\
\hline \multirow[t]{2}{*}{$50-54$} & Feminino & $\ldots$ & & & $y=-211,4+2,4 x$ & 85 & $<0,001$ \\
\hline & Masculino & $y=-71,5+0,8 x$ & 60 & 0,001 & $y=-8.966,5+194,3 x-1,0 x^{2}$ & 79 & $<0,001$ \\
\hline \multirow[t]{2}{*}{$55-59$} & Feminino & $\ldots$ & & & $y=-95,0+1,1 x$ & 78 & $<0,001$ \\
\hline & Masculino & $\ldots$ & & & $y=-4.697,8+102,2 x-0,6 x^{2}$ & 81 & $<0,001$ \\
\hline \multirow[t]{2}{*}{$60+$} & Feminino & $\ldots$ & & & $y=-122,7+1,4 x$ & 87 & $<0,001$ \\
\hline & Masculino & $\ldots$ & & & $y=-5.350,0+116,3 x-0,6 x^{2}$ & 85 & $<0,001$ \\
\hline
\end{tabular}

*Modelo: $\mathrm{y}=$ número de casos de AIDS e $\mathrm{x}=$ (ano do diagnóstico - 1991). ... : ausência ou número muito pequeno de casos

*Model: $y=$ number of AIDS cases $; x=$ (year of diagnosis - 1991). ... : no cases or small number of AIDS cases

UDI, com a única exceção do grupo de 15 a 19 anos. A variação do crescimento médio anual foi em torno de 13 casos, na faixa etária de 25 a 34 anos, a mais atingida, até um caso em mulheres com idade acima de 54 anos (Tabela 2).

Já para os homens UDI, as tendências nas faixas etárias inferiores aos 45 anos foram sempre de crescimento no início da epidemia, passando a decrescer a partir de 1992. Nas faixas etárias de 45 a 49 e 50 a 54 anos as tendências foram de crescimento constante ao longo de todo o período de es- tudo (respectivamente, 2,2 e 0,8 casos ao ano).

As curvas de tendência para os homens não UDI em todas as faixas etárias foram semelhantes: crescentes inicialmente, passando a decrescer a partir de 1992 a 1994, conforme a grupo etário.

Analisando separadamente a tendência dos casos de AIDS em homens não UDI heterossexuais e HSH, observou-se que na faixa etária a partir de 20 anos houve tendência de declínio para HSH com $\mathrm{R}^{2}$ de $90 \%$ e $\mathrm{p}<0,001\left(\mathrm{y}=-141 \cdot 122,0+3.082,0 \mathrm{x}-16,7 \mathrm{x}^{2}\right)$, 
mas para heterossexuais o declínio somente ocorreu na faixa de 20 a 24 anos ( $y=-3.373,8$ $+72,5 \mathrm{x}-0,4 \mathrm{x}^{2} ; \mathrm{R}_{\mathrm{a}}^{2}$ de $\left.63 \% ; \mathrm{p}=0,002\right)$, enquanto na faixa a partir de 25 anos houve crescimento de 46 casos ao ano ( $\mathrm{y}=-3.919,5$ $+45,9 \mathrm{x} ; \mathrm{R}_{\mathrm{a}}^{2}$ de $\left.89 \% ; \mathrm{p}<0,001\right)$.

\section{Discussão}

Analisar a epidemia de AIDS através dos casos é estudar o passado da epidemia de pelo menos 5 a 10 anos, e construir uma imagem que permita observar trajetórias e processos, pois não é possível apreender todas as faces do acontecido. Citando MerleauPonty ${ }^{24}$ "Não se trata de coincidir com o que foi vivido: trata-se de decifrar o sentido total daquilo que foi feito" (p. 5).

Os pacientes UDI participaram com cerca de um terço dos casos estudados, mas as curvas de tendência da epidemia verificada em ambos os grupos foram semelhantes, observando-se um declínio a partir de 1993, ainda que mais acentuado entre os primeiros (Figura 1-A). Essa mesma tendência de declínio foi observada por Moraes e colaboradores ${ }^{25}$ para os casos de AIDS no Estado de São Paulo, através da análise dos casos notificados até 1995.

Ainda que o retardo nas notificações, especialmente as relativas aos últimos 12 meses ${ }^{26}$, subestime os dados referentes ao final do período, esta influência foi minimizada no presente estudo, devido à utilização dos casos notificados até setembro de 1998.

A epidemia de AIDS em UDI apresentou uma ascensão importante a partir de 1987, elevação que pareceu refletir em parte o aumento na sensibilidade do sistema de informação decorrente da nova definição. Fato semelhante foi descrito nos Estados Unidos ${ }^{27}$, onde essa nova definição de caso foi responsável por aumento de casos confirmados entre UDI proporcionalmente maior do que entre outras categorias de exposição.

Em 1992, houve o pico da incidência da AIDS no Município de São Paulo, coincidindo com nova alteração da definição de caso $^{28}$; o aumento do número total de casos notifi- cados nesse ano, em relação a 1991, foi de $22,8 \%^{2}$, com estabilização em patamares semelhantes a 1992 nos dois anos seguintes. Essa modificação da definição de caso, provavelmente explica, ao menos em parte, o aumento de casos confirmados de AIDS tanto para UDI quanto para não UDI, respectivamente de $11,7 \%$ e $28,8 \%$. Dentre os casos notificados entre não UDI, houve aumento de 65,8\% para heterossexuais, em relação ao ano anterior, com maior proporção para o sexo feminino $(79,4 \%)$ do que para o masculino (56,8\%).

As sucessivas mudanças de definição de caso constituem um fator limitante para análise de tendência de AIDS, pois provocam artificialmente aumentos bruscos da incidência, fato observado em diversos países como a Dinamarca $^{29}$, Espanha $^{30}$ e Estados Unidos ${ }^{31}$, com maior identificação de casos entre certas categorias de exposição como UDI, como ocorreu nos dois últimos países citados ${ }^{30,31}$, ou ainda, maior inclusão de mulheres como na Dinamarca ${ }^{29} \mathrm{e}$ nos Estados Unidos ${ }^{31}$.

A tendência da epidemia de AIDS em UDI, tomando como referência os casos notificados, apresenta declínio a partir de 1993, abrangendo tanto HSH como os heterossexuais de ambos os sexos, nas faixas etárias de 15 a 34 anos, nas mulheres e de 15 a 44 anos, nos homens.

O declínio da incidência de AIDS também foi observado em áreas de alta prevalência inicial de infecção por HIV entre UDI, como Nova Iorque, Nova Jérsei ${ }^{32}$ e Itália ${ }^{33}$. Tal declínio, provavelmente, deveu-se a convergência de vários fatores, como as mudanças de comportamento, a redução de suscetíveis entre os UDI e a utilização de terapias anti-retrovirais entre infectados pelo HIV, em período anterior à manifestação da doença. Tais fatores contribuíram para a queda da incidência da AIDS observada na Europa Ocidental ${ }^{34}$ e nos Estados Unidos ${ }^{35}$, no período de 1990 a 1995.

Alem da alteração de comportamento de compartilhar equipamentos de injetar e preparar a droga ${ }^{36}$, a mudança da via de administração também pode ser responsável pela queda no número de casos ou da soropre- 
valência. A ameaça da AIDS, de modo geral, mudou o comportamento dos UDI; isso teria ocorrido em São Paulo, conforme relatado por Dunn e colaboradores ${ }^{37}$ e por Fernandez ${ }^{38}$, pelo aumento do consumo do crack, a partir de 1990, e redução do uso de cocaína injetável.

A diminuição dos suscetíveis entre os expostos é uma hipótese a ser considerada para explicar a diminuição da incidência observada $^{39}$; no entanto, tal possibilidade deve ser considerada com cuidado, pois a população é heterogênea em relação ao risco, apresentando diferentes graus de exposição e, em conseqüência, diferentes proporções de suscetíveis. Neste sentido, o declínio não aponta, obrigatoriamente, uma tendência para os próximos anos e pode significar somente uma oscilação temporária ${ }^{39}$.

Novos picos na epidemia poderão ocorrer pela disseminação da epidemia entre grupos com grande número de suscetíveis, devido a não manutenção de comportamento seguro em decorrência de recaídas no uso de drogas injetáveis ${ }^{40}$, compartilhando equipamentos para o preparo e uso das mesmas, ou pela alternância entre vias de administração, que faz parte da subcultura da droga ${ }^{41}$, além das práticas de sexo não seguro.

Não há dados suficientes a respeito de um possível declínio na infecção pelo HIV entre UDI, mas se pode ousar dizer que os níveis de soroprevalência possivelmente permanecem altos, pois isso ocorre em outros países $^{32,33}$, demonstrando que mudanças de comportamento ainda são fundamentais para reduzir a transmissão.

Para o controle da epidemia em UDI é indispensável que os Programas de Prevenção sejam voltados tanto para os adolescentes quanto para os adultos jovens, pois há grande proporção de pessoas UDI com AIDS na faixa etária entre 20 a 34 anos, em ambos os sexos, provavelmente, infectadas por volta dos 14 aos 28 anos (admitindo um período de incubação mínimo de 6 anos). Boa parte dos UDI parece que se tornam soropositivos para o HIV ainda na adolescência, por compartilharem equipamentos de preparo e apli- cação de drogas injetáveis com UDI que pertencem a redes de alto risco para à infecção, assim como pela prática de sexo não seguro. Vale lembrar que, neste estudo, observouse a tendência da epidemia se manter constante na faixa etária de 15 a 19 anos.

Nas faixas etárias a partir de 35 anos entre UDI, para as quais foi possível realizar a modelagem, notou-se que a tendência para UDI foi diferente, se comparada com a faixa etária mais jovem. Mas este é, provavelmente, um efeito de coorte em que as pessoas infectadas pelo HIV no início da epidemia apresentaram um período de incubação mais longo se comparados aos infectados mais recentemente, efeito também observado por Hamers e colaboradores ${ }^{34}$ na epidemia em UDI na Europa.

Considerando todos os aspectos já relatados, parece ter havido um declínio real da epidemia de AIDS em UDI no Município de São Paulo, mas sua vulnerabilidade ao HIV ainda permanece.

A tendência da epidemia de AIDS em não UDI apresentou, a partir de 1993, uma evolução um tanto distinta daquela verificada entre os UDI, com declínio menos acentuado do que esta e estabilização em patamares elevados.

Essa diferença decorre da tendência verificada entre heterossexuais não UDI que mostrou inclinação crescente com aumento médio anual de 107 casos, enquanto que o declínio entre HSH UDI foi evidente.

As mulheres não UDI contribuíram com $13,5 \%$ do total de casos e a tendência nesse grupo foi continuamente ascendente com crescimento médio anual de 59 casos. Essa tendência se repete para todas as faixas etárias. O crescimento da incidência nessa parcela da população com grande número de pessoas suscetíveis ao HIV ocorreu provavelmente por dois fatores.

Um deles foi o retardo no diagnóstico e por conseqüência da introdução de terapias anti-retrovirais, decorrente da menor ênfase conferida no início da epidemia ao controle da AIDS entre as mulheres. Merece atenção o fato da maioria das mulheres não UDI estudadas terem possivelmente se 
infectado entre os 14 e 28 anos de idade (admitindo um período de incubação mínimo de 6 anos), indicando início precoce da vida sexual sem orientação adequada.

Outro fator importante para a disseminação da epidemia foi a prática de sexo não seguro entre mulheres não UDI e homens UDI ou homens não UDI com múltiplas parceiras sexuais. Isso pode ser parcialmente visualizado pelo fato de 40,5\% (574/1417) das mulheres não UDI com AIDS relatarem como único parceiro uma pessoa UDI, o mesmo acontecendo com $41,5 \%$ das pacientes notificadas (1417/3414). O contínuo aumento da epidemia devido a transmissão por meio de relações sexuais entre pessoas UDI e não UDI, também foi observado nos Estados Unidos ${ }^{42}$.

Mas o impacto desse efeito para a epidemia em São Paulo ainda é possivelmente subestimado, pois para mais de $50 \%$ das mulheres não UDI as informações sobre os parceiros eram desconhecidas, à semelhança do verificado na Europa onde $40 \%$ desconheciam o fator de risco que fez com que seus parceiros se infectassem ${ }^{34}$. Os resultados do presente estudo e o que foi observado nos Estados Unidos ${ }^{42}$ e Europa ${ }^{34}$ sugerem um aumento da vulnerabilidade feminina.

Vale salientar que à semelhança das mulheres não UDI, os homens heterossexuais não UDI com AIDS também apresentaram grande proporção de informação ignorada quanto a parceria sexual (acima de 55\%), ao contrário dos HSH com AIDS que demonstram ter grande conhecimento sobre o parceiro.

A maioria dos HSH não UDI negaram que o parceiro tenha tido múltiplos parceiros ou que seria soropositivo ou usuário de droga injetável e 95\% deles informaram que eles próprios não tiveram múltiplos parceiros. $\mathrm{O}$ grau de informação a respeito do parceiro, a mudança de comportamento sexual e o acesso às terapias anti-retrovirais são o reflexo de ações preventivas voltadas para esse grupo populacional que, provavelmente, contribuíram para a redução de sua vulnerabilidade, pois a tendência da epidemia nessa parcela da população foi de declínio.

A tendência da epidemia de AIDS entre os homens que informaram serem heteros- sexuais e não UDI foi crescente e diferente da dos HSH. Essa diferença entre as tendências e a relação de 1:1 entre homens e mulheres heterossexuais não UDI não sugeriu a existência de erro de classificação entre heterossexuais e HSH, como observado por Castilho e colaboradores ${ }^{43}$, exceto para homens entre 20 e 24 anos que informaram parceria heterossexual, mas a tendência mostrou-se semelhante à de HSH.

Entre os fatores limitantes dos dados analisados, um é a sensibilidade do sistema de vigilância. Avaliação do sistema de vigilância da AIDS no Município de São Paulo efetuada por Drumond Jr e colaboradores ${ }^{44}$, tomando como parâmetro para o cálculo da sensibilidade o número de óbitos por AIDS, verificou que o mesmo só consegue captar $85 \%$ dos casos da doença que evoluíram para óbito. Pode-se citar, também, como limitações do estudo, a possibilidade de erros no registro da idade, na classificação do tipo de exposição, pela heterogeneidade das fontes de informação e pelo caráter íntimo da informação solicitada aos pacientes com AIDS. Vale registrar também que, nos últimos anos da série, a proporção de casos de AIDS com categoria de exposição "ignorada" aumentou progressivamente, situando-se acima de 30\% a partir de 1994.

Outro fator de erro decorre da classificação dos casos com informação exclusiva de uso de droga injetável, e "relação sexual ignorada", como heterossexuais. Isso pode interferir na análise de homens heterossexuais UDI, pois essa definição operacional aumentou em $23,1 \%$ os casos nessa categoria.

A associação entre exposição por contato heterossexual e transfusão de sangue e hemoderivados foi estatisticamente significativa em relação a HSH, mas não houve diferença entre UDI e não UDI. Portanto, entre os heterossexuais pode ter existido outra exposição a sangue que não o uso de droga injetável, mas a possibilidade de que isso tenha ocorrido é semelhante entre UDI e não UDI.

O retardo na implantação do Programa de Redução de danos na cidade de São Paulo deve ter influenciado a trajetória da epidemia 
de AIDS entre UDI e mulheres. No entanto, mesmo com sua implantação, ainda existe o desafio de conhecer e ter acesso à maioria da população de UDI, a fim de que se possa evitar novo aumento de casos nesse grupo.

Alguns trabalhos facilitam a melhor compreensão da epidemia de AIDS entre os UDI. Segundo Kalichman ${ }^{13}$, no Município de São Paulo somente 0,9\% dos UDI apresentava nível universitário, contrastando com 22,5\% entre os HSH e 6,2\% entre os heterossexuais. Além disso, 49\% dos UDI e 52,6\% dos heterossexuais viviam em áreas pobres, enquanto 53,6\% dos HSH residiam em áreas ricas. Grangeiro ${ }^{45}$, analisando a ocupação das pessoas com AIDS, observou que grande parte dos UDI estavam em ocupações não especializadas ou desempregados e que $55 \%$ dos ajudantes gerais ou serventes eram UDI.

Para conhecer a dinâmica da epidemia na cidade é preciso compreender as redes que se formam a partir da subcultura da droga e as possibilidades de mistura entre redes com diferentes riscos para a infecção HIV, através de uma abordagem antropológica, assim como estudos de redes sociais, com análises quantitativas de sociodramas.

Muitos desafios são colocados para o controle da epidemia no Município como: a manutenção dos recursos para o Programa de Trocas de Seringas (PTS); a implantação completa do Programa de Redução de Danos, com o oferecimento de tratamento para dependência química, na proporção de sua importância para a cidade, e a abordagem nas ruas e escolas de potenciais usuários de drogas; assim como fazer com que o discurso preventivo transforme-se em prática preventiva para as mulheres e os homens da cidade.

Esse estudo não pretendeu compreender totalmente este complexo fenômeno, mas tornar visível parte dele. Os dados utilizados formaram uma imagem da epidemia em UDI e não UDI no Município de São Paulo e deram um panorama da história da epidemia.

\section{Agradecimentos}

A Prof ${ }^{a}$ Maria do Rosário Dias de Oliveira Latorre agradece ao CNPq a bolsa de produtividade em pesquisa (processo no 30 0318/97-9).

\section{Referências}

1. Ministério da Saúde. Secretaria de Políticas de Saúde Coordenação Nacional de DST e AIDS. Bol Epidemiol AIDS 2001; 14 (2):7-14.

2. Secretaria de Estado da Saúde de São Paulo. Centro de Referência e Treinamento DST/AIDS. Bol Epidemiol Prog DST/AIDS 2001; 19(1): 35-9.

3. Carvalho HB, Mesquita F, Massad E, Bueno RC, Lopes GT, Ruiz MA, Burattini MN. HIV and infections of similar transmission patterns in a drug injectors community of Santos, Brazil. J AIDS Hum Retrovirol 1996; 12: 84-92.

4. Andrade T, Dourado I, Galvão-Castro B. Associations among HTLV-1, HTLV-II and HIV in injecting drug users in Salvador, Brazil. J AIDS Hum Retrovirol 1998; 18: 186-91.

5. Grangeiro A, Kalichman AO, Guibu IA, Jamal JF, Santos NJS, Teixeira PR, Munhoz R. AIDS e drogas no estado de São Paulo. Bol Epidemiol Prog DST/AIDS 1996; 3: 2-10.
6. Telles PR, Bastos FI, Guydish J, Inciardi JA, Surratt HL, Pearl M, Hearst N. Risk behavior and HIV soroprevalence injecting drug users in Rio de Janeiro, Brazil. AIDS 1997; 11 Suppl 1: S35-S42.

7. World Health Organization. An international comparative study of HIV prevalence and risk behaviour among drug injectors in 13 cities. Bull Narc 1993; 45: 19-46.

8. Des Jarlais DC, Friedman SR, Perlis T, Chapman TF, Sotheran JL, Paone D et al. Risk behavior and HIV infection among new drug injectors in the era of AIDS New York City. J AIDS Hum Retrovirol 1999; 20: 67-72.

9. Garfein RS, Vlahov D, Galai N, Doherty MC, Nelson KE. Viral infections in short-term injection drug users: the prevalence of the hepatitis $C$, hepatitis $B$, human immunodeficiency and human T-lymphotropic viruses. Am J Public Health 1996; 86: 655-61. 
10. Rhodes T, Bueno R, Myers T, Hunter GM, Stimson GV. Cross-national differences in sexual risk behaviour among cocaine and opioid IDUs in Santos, London and Toronto: prevention implications. In: Abstracts of the $11^{\text {th }}$ International Conference on AIDS; 1996 Jul 7-12; Vancouver (CA). Vancouver: WHO; 1996. v 1, p.352 [Tu.C.2506].

11. Bastos FI, Barcellos C, Lowndes CM, Friedman SR. Coinfection with malaria and HIV in injecting drug users in Brazil: a new challenge to public health? Addiction 1999; 94: 1165-74.

12. Casseb J, Caterino-de-Araujo A, Hong MA, Salomão S, Gallo D, Hendry RM, Duarte AJS. Prevalence of HTLV-1 and HTLV-II infections among HIV-1- infected asymptomatic individuals in São Paulo, Brazil. Rev Inst Med Trop São Paulo 1997; 39: 213-5.

13. Kalichman AO. AIDS and intravenous drug use (IVUD) in Brasil. In: Monteiro MG, Inciardi JA, editors.

Proceedings of the Brazil - United States: Binational Meeting on Drug Abuse Research; 1992 May 21-23; São Paulo (Brazil). São Paulo: CEBRID; 1992. p.50-61.

14. Newmeyer JA. The intravenous drug and secondary spread of AIDS. J Psychoactive Drugs 1988; 20: 16972.

15. Barcellos C, Bastos FI. Redes sociais e difusão da AIDS no Brasil. Bol Oficina Sanit Panam 1996; 121: 11-24.

16. Centers for Disease Control and Prevention. Revision of the case definition of acquired immunodeficiency syndrome for national reporting - United States. MMWR Morb Mortal Wkly Rep 1985; 34: 373-5.

17. Centers for Disease Control and Prevention. Revision of the CDC surveillance case definition for acquired immunodeficiency syndrome - leads from the MMWR (suppl 1S). JAMA 1987; 258: 1143-54.

18. Ministério da Saúde. Programa Nacional de DST/AIDS. Revisão da definição nacional do caso de AIDS em adultos. Brasília (DF); 1992.

19. Secretaria de Estado da Saúde São Paulo. Coordenação dos Institutos de Pesquisa. Centro de Referência e Treinamento DST/AIDS. Ofício 07/97. São Paulo; 1997.

20. Ministério da Saúde. Coordenação Nacional de DST/ AIDS. Bol Epidemiol AIDS 1998; 11: 8-40.

21. SPSS for Windows 8.0.0. [computer program]. SPSS Inc.; 1997.

22. Latorre MRDO. A mortalidade por câncer de estômago no Brasil: análise do período de 1977 a 1989. Cad Saúde Pública 1997; 13 Supl 1: 67-78.

23. Kleinbaum DG, Kupper LL, Muller KE. Aplied regression analysis and other multivariable methods. $2^{\text {nd }}$ ed. Belmont: Duxbury; 1988.
24. Merleau-Ponty M. Textos selecionados: Maurice Merleau-Ponty. Trad. de MS Chauí. São Paulo: Nova Cultural; 1989. As aventuras da dialética: a crise do entendimento; p.3-20. (Os Pensadores).

25. Moraes JC, Seabra NSS, Eluf Neto J. São Paulo. In: Ministério da Saúde. Simpósio Satélite A Epidemia da AIDS no Brasil: Situação e Tendências [on line]. Brasília (DF): Ministério da Saúde; 1997. Disponível em <URL: http://www.aids.gov.br>. [1999 out 10].

26. Bandeira ACA. Estimativa do número de pessoas infectadas com o vírus HIV e projeção de casos novos de AIDS para o município de São Paulo através do método da retroestimativa ("backcalculation") [Dissertação de Mestrado]. São Paulo: Faculdade de Medicina da USP; 1995.

27. Centers for Disease Control and Prevention. Update: acquired immune deficiency syndrome associated with intravenous drug use United States, 1988. MMWR Morb Mortal Wkly Rep 1989; 38: 165-70.

28. Bandeira ACA, Ribeiro AF. O impacto da nova classificação de AIDS em adultos (critério Caracas) no Município de São Paulo em 1993. In: Resumos dos trabalhos do $3^{\circ}$ Congresso Brasileiro de Epidemiologia; 1995 abr 24-28; Salvador, Brasil. Salvador: Associação Brasileira de Saúde Coletiva; 1995. p. 260

29. Smith E, Mathiesen L, Skinhoj P, Seefeld T, Isager H. Impact of the 1987 revised AIDS case definition in Denmark: a follow-up study 2 years after its adoption. Scand J Infect Dis 1992; 24: 293-9.

30. Casabona J, Salas T, Segura A. The impact of the revised case definition of AIDS. JAMA 1988; 260: 2213.

31. Selik RM, Buehler JW, Karon JM, Chamberland ME, Berkelman RL. Impact of the 1987 revision case definition of acquired immune deficiency sindrome in the United States. J Acquir Immune Defic Syndr 1990; 3: 73-82.

32. Green TA, Karon JM, Nwanyanwu OC. Changes in AIDS incidence trends in the United States. J Acquir Immune Defic Syndr 1992; 5: 547-55.

33. Rezza G, Nicolosoi A, Zaccarelli M, Sagliocca L, Nespoli M, Gattari P et al. Understanding the dynamics of the HIV epidemic among italian intravenous drug users: a cross-sectional versus a longitudinal approach. $J$ Acquir Immune Defic Syndr 1994; 7: 500-3.

34. Hamers FF, Batter V, Downs AM, Alix J, Cazein F, Brunet JB. The HIV epidemic associated with injecting drug use in Europe: geographic and time trends. AIDS 1997; 11: 1365-74.

35. Gail MH, Rosenberg PS, Goedert JJ. Therapy may explain recent deficits in AIDS incidence. J Acquir Immune Defic Syndr 1990; 3: 296-306.

36. Des Jarlais DC. Cross-national studies of AIDS among injecting drug users. Addiction 1994; 89: 383-92. 
37. Dunn J, Laranjeira RR, Silveira DX, Formigoni MSOS, Ferri CP. Crack cocaine: an increase in use among patients attending clinics in São Paulo: 1990-1993. Subst Use Misuse 1996; 31: 519-27.

38. Fernandez OFRL. A epidemia clandestina: AIDS e usuários de drogas endovenosas em São Paulo. [Dissertação de Mestrado]. São Paulo: Pontifícia Universidade Católica de São Paulo; 1993.

39. Anderson RM. The spread of HIV and sexual mixing patterns. In: Mann J, Tarantola DJM, editors. AIDS in the world II: global dimensions, social roots, and responses. New York: Oxford University Press; 1996. p.71-86.

40. Des Jarlais DC, Abdul-Quader A, Tross S. The next problem: maintenance of AIDS risk reduction among intravenous drug users. Int J Addict 1991; 26: 1279-92.

41. Des Jarlais DC, Friedman SR, Choopanya K, Vanichseni S, Ward T. International epidemiology of HIV and AIDS among injecting drug users. AIDS 1992; 6: $1053-68$.
42. Neal JJ, Fleming PL, Green TA, Ward JW. Trends in heterosexually acquired AIDS in United States, 1988 through 1995. J AIDS Hum Retrovirol 1997; 14: 46574.

43. Castilho E et al. Features of heterosexual exposure category in Brazil, 1980-1990. In: Abstracts of the $7^{\text {th }}$ International Conference on AIDS; 1991; Florence (Italy). Florence; 1991 p. 361. [Mc 3252].

44. Drumond Júnior M, Lira MMTA, Freitas M, Nitrini TMV. A AIDS e os sistemas de informação de mortalidade em nível local: a experiência do PRO-AIM no Município de São Paulo. Secretaria de Estado da Saúde de São Paulo. Centro de Referência e Treinamento DST/AIDS. Bol Epidemiol Progr DST/AIDS 1997; 15: 3-9.

45. Grangeiro A. O perfil socioeconômico dos casos de AIDS na cidade de São Paulo. In: Parker R et al., organizadores. A AIDS no Brasil. Rio de Janeiro: Relume-Dumará; 1994. p.91-125. (História Social da AIDS, 2). 